\title{
Structure of spaces of rhombus tilings in the lexicograhic case
}

\author{
Eric Rémila ${ }^{1,2}$ \\ ${ }^{1}$ Laboratoire de l'Informatique du Parallélisme (umr 5668 CNRS-INRIA-Univ. Lyon 1-ENS Lyon), \\ Ecole Normale Supérieure de Lyon, 46 allée d'Italie, 69364 Lyon Cedex 07, France \\ ${ }^{2}$ IUT Roanne (Univ. Saint-Etienne), \\ 20 avenue de Paris, 42334 Roanne Cedex, France
}

Rhombus tilings are tilings of zonotopes with rhombohedra. We study a class of lexicographic rhombus tilings of zonotopes, which are deduced from higher Bruhat orders relaxing the unitarity condition.

Precisely, we fix a sequence $\left(v_{1}, v_{2}, \ldots, v_{D}\right)$ of vectors of $\mathbb{R}^{d}$ and a sequence $\left(m_{1}, m_{2}, \ldots, m_{D}\right)$ of positive integers. We assume (lexicographic hypothesis) that for each subsequence $\left(v_{i_{1}}, v_{i_{2}}, \ldots, v_{i_{d}}\right)$ of length $d$, we have $\operatorname{det}\left(v_{i_{1}}, v_{i_{2}}, \ldots, v_{i_{d}}\right)>0$. The zonotope $Z$ is the set $\left\{\sum \alpha_{i} v_{i} 0 \leq \alpha_{i} \leq m_{i}\right\}$. Each prototile used in a tiling of $Z$ is a rhombohedron constructed from a subsequence of $d$ vectors.

We prove that the space of tiilngs of $Z$ is a graded poset, with minimal and maximal element.

Keywords: rhombus tiling, flip, connectivity

\section{Introduction}

Rhombus tilings are tilings of zonotopes with rhombohedra. They appear in physics as a classical model for quasicrystals (8). We fix a sequence $\left(v_{1}, v_{2}, \ldots, v_{D}\right)$ of vectors of $\mathbb{R}^{d}$ (such that each subsequence of length $d$ is a basis of $\left.\mathbb{R}^{d}\right)$ and a sequence $\left(m_{1}, m_{2}, \ldots, m_{D}\right)$ of positive integers (called multiplicities). The tiled zonotope $Z$ is the set $\left\{\sum \alpha_{i} v_{i} 0 \leq \alpha_{i} \leq m_{i}\right\}$, and each prototile used for $T$ is a rhombohedron constructed from a subsequence of vectors of length $d$. If a tiling $T$ contains $d+1$ rhombic tiles which pairwise share a facet, then a new tiling $T_{f l i p}$ of $Z$ can be obtained just changing the position of those $d+1$ tiles. This operation is called a flip. The space of tilings of $Z$ is the graph whose vertices are tilings of $Z$ and two tilings are linked by an edge if they differ by a single flip.

Before this paper, study has been done by Ziegler (9), about higher Bruhat orders. Those combinatorial structures can be interpreted (via the Bohne-Dress theorem (7)) as tilings of some specific unitary zonotopes (i.e. all multiplicities are equal to 1). Ziegler proves that, this case in this the space of tilings can be directed so as to get a graded poset (with single maximal and minimal element).

In the present paper, we extend the previous result relaxing the unitarity condition. We first recall how ideas (deletion, minors) issued from matroid theory to get a decomposition method for tilings, and a representation of tilings by black or white points organized in arrows and lines (see $(2 ; 3)$ for details). Afterwards, we use this representation to study that we call lexicographic tilings (extensions with non 


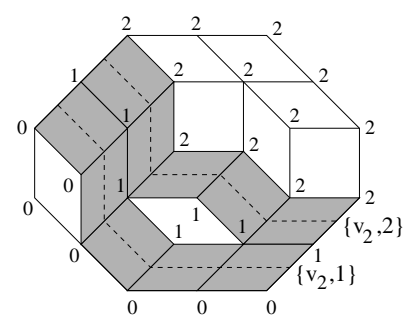

Fig. 1: The 2-located height function and two de Bruijn sections

unitary multiplicities of tilings correspondings to higher Bruhat orders ) We show how each space of lexicographic tilings can be directed so as to get a graded poset (with single maximal and minimal element), which implies the connectivity of the space. All technical details can be found in (6).

About related works, Felsner and Weil (4) prove the same result, when $d=2$. To our knowledge, the connectivity problem is still open for the other kinds of zonotopes. We mention that R. Kenyon (5) has proved the connectivity in dimension 2 , for any simply connected domain.

\section{Rhombus tilings, minors, and representation}

Let $V=\left(v_{1}, \ldots, v_{D}\right)$ be a sequence of vectors in $\mathbb{R}^{d}$ such that $D \geq d$ and each subsequence $\left(v_{i_{1}}, v_{i_{2}}, \ldots, v_{i_{d}}\right)$ is a basis of $\mathbb{R}^{d}$ and $M=\left(m_{1}, \ldots, m_{D}\right)$ be a sequence of $D$ positive integers. The parameter $c=D-d$ is the codimension, the integer $m_{i}$ is called the multiplicity of $v_{i}$. The zonotope $Z(V, M)$ is the region of $\mathbb{R}^{d}$ defined by: $\left\{v \in \mathbb{R}^{d}, v=\sum_{i=1}^{D} \lambda_{i} v_{i},-m_{i} \leq \lambda_{i} \leq m_{i}\right\}$. The region $Z(V, M)$ is a finitely generated convex set. One can define classically (see for example (10) p. 51-52) its faces, vertices, edges and facets. The number: $s=\sum_{i=1}^{D} m_{i}$ is the size of the zonotope $Z$; we say that $Z$ is a s-zonotope. $Z$ is said to be unitary if all the multiplicities are equal to 1 . A prototile is a unitary zonotope constructed with a subsequence $V^{\prime}$ of $d$ distinct vectors taken in $V$. A tile $t$ is a translated prototile. A tiling $T$ of a zonotope $Z(V, M)$ is a set of tiles such that each intersection between tiles is a face and the union of all tiles is $Z(V, M)$ Two tiles are adjacent if they share a whole facet. We say that $Z(V, M)$ is the support of the tiling $T$. A unitary zonotope of codimension 1 admits exactly two symmetrical tilings: one of these small tilings can appear, up to translation, in a tiling $T$ of a larger zonotope $Z$. in this case, one can replace it by the other tiling, to obtain another tiling $T^{\prime}$ with the same support . This operation is called a flip. The space of tilings of $Z$ is the symmetric graph whose vertices are tilings of $Z$, and two tilings are linked by an edge if they differ by a flip. Each edge is labeled by the sequece of vectors of the flip

For each integer $k$ such that $1 \leq k \leq D$, and each tiling $T$ of $Z(V, M)$, a $k$-located height function $h_{T, k}$ is a function such that, for any pair $\left(x, x^{\prime}\right)$ of vertices of $T$ such that $x^{\prime}=x+2 v_{i}$ and $\left[x, x^{\prime}\right]$ is an edge of $T, h_{T, k}\left(x^{\prime}\right)=h_{T, k}(x)+1$ if $i=k$ and $h_{T, k}\left(x^{\prime}\right)=h_{T, k}(x)$ otherwise. We use the normalized $k$-located height function such that, for each vertex $x, h_{T, k}(x) \leq 0$ and there exists a vertex $x_{0}$ such that $h_{T, k}\left(x_{0}\right)=0$

The de Bruijn zone $S_{\left\{v_{i}, j\right\}}$ of $T$ is the set of tiles whose normalized $i$-located function is $j-1$ on one facet, and $j$ on the opposite one. See Figure 1. A de Bruijn zone $S_{\left\{v_{i}, j\right\}}$ disconnects the tiling into two parts. One can remove the tiles of $S_{\left\{v_{i}, j\right\}}$ and translate all the tiles of one of these parts. For $D>d$, the configuration obtained is a tiling of another zonotope $Z^{\prime}=\left(V, M^{\prime}\right)$. Such an operation is called deletion. 
The tiling obtained is denoted by $D_{\left\{v_{i}, j\right\}}(T)$. A tiling obtained from $T$ by a sequence of $p$ deletions is called a $(s-p)$-minor of $T$. The pairs $\left\{v_{i}, j\right\}$ can be totally ordered (by the lexicographic order, for example). From this order, the sets formed by $p$ elements of the type $\left\{v_{i}, j\right\}$ can also be totally ordered Therefore, the $(s-p)$-minors of $T$ can be totally ordered. The sequence of $(s-p)$-minors of $T$ is given by this order.

For $s \geq d+2$, every tiling $T$ of $Z(V, M)$ is defined by the sequence of its $d+1$-minors. The free $d+1$-minors are tilings of unitary $d+1$-zonotopes. They contain some information, useful to compute $T$, which can be reduced to a single bit (since each unitary $d+1$-zonotope has exactly two tilings) See Figure 2. Let $T$ and $T^{\prime}$ be two tilings of a same zonotope. One can prove that their $d+1$-minors, except one, are the same if and only if they differ by a flip. Conversely; given a zonotope $Z$ and a sequence of $d+1$-tilings, does there exist a tiling $T$ of $Z$ such that the given sequence is the sequence of its $d+1$ minors ? The problem can be solved by constructing the (potential) $d+2$-minors, then the $d+3$-minors, and so on until the D-tiling is found. But the answer can be given faster: the first step is enough to obtain the answer.

Proposition 1 (2) Let $(Z m)_{m}$ be a sequence of codimension 1 tilings. There exists a tiling $T$ of a zonotope $Z$ whose sequence of $d+1$-minors is exactly $(Z m)_{m}$ if and only if the $d+2$-minors are compatible, i.e. can be correctly constructed.

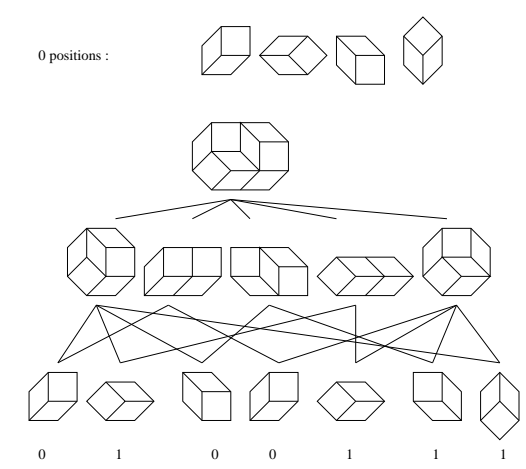

Fig. 2: Coding a tiling with minors.

If we have $D$ vectors, we arbitrarily fix a basic tiling $T_{0}$ of the unitary $D$-zonotope. For each $d+1$ unitary zonotope, we define the low position as the $d+1$-tiling of this zonotope which is a $d+1$-minor of $T_{0}$. The other $d+1$-tiling is the high position. With this definition, flips can be canonically directed: a flip is going upwards if it transforms a low position in a high position. The directed space of tilings is the space of tilings whose edges are directed as above. this graph $\mathrm{t}$ is acyclic and, therefore, defines a partial order relation, denoted by $<_{\text {flip }}$.

In dimension $d$, there exists two basic kinds of $d+2$-zonotopes of dimension $d$ whose tiling is not forced: either all vectors have multiplicity 1 (codimension 2), or there is one vector of multiplicity 2 (codimension 1). The directed space of tilings of the zonotope $Z_{i}$ of codimension 1 with the vector $v_{i}$ of multiplicity 2 (and the $d$ other ones of multiplicity 1) contains three tilings and is a chain of length 2 . The space of tilings of a unitary $d+2$-zonotope is a cycle of length $2(d+2)$, and each possible label is given 
to a pair of edges, which are opposite in the cycle. The directed space is formed by two chains of length $d+2$ which only meet on endpoints. We extensively use the previous examples to represent tilings

Points: In our representation, each $d+1$-minor is associated to a point. Each point $p$ is given a color, which is white if the $d+1$-minor is in low position, or black if in high position. The important thing for reconstructing a tiling $T$ is the set of coloring constraints (which are given by the sequence of $d+2$-minors). We now explain how coloring constraints are expressed.

Lines: Now, consider a $d+2$-minor of $T$ whose support is a unitary $d+2$-zonotope. The $d+2 d+1$ minors of the $d+2$-minor can be ordered on a chain. From what has been seen about tilings of unitary $d+2$-zonotopes, with the order convention, the black points have to form a final or initial segment (i. e. a suffix or a prefix) of the line. This is the line constraint.

With this tool, spaces of tilings with unitary zonotopes can be represented by sets of lines with black and wihite points, as it can be classically obtained using with matroid duality and the topological representation theorem (1). In order to represent tilings for any zonotope, we have introduced some arrows.

Arrows: Choose two points corresponding to the pair of minors of a same $d+2$-minor of $T$ (whose support is a $d+2$-zonotope of codimension 1). There exists exactly three allowed colorings corresponding to tilings of this $d+2$-zonotope. In our representation, an arrow is placed, linking these two points, in such a way that the three allowed colorings of the tiled $d+2$-zonotope are the fully black one, the fully white one, and the coloring with the origin of the arrow being black and the tail being white. This gives the arrow constraint: there is no edge from a white point to a black point.

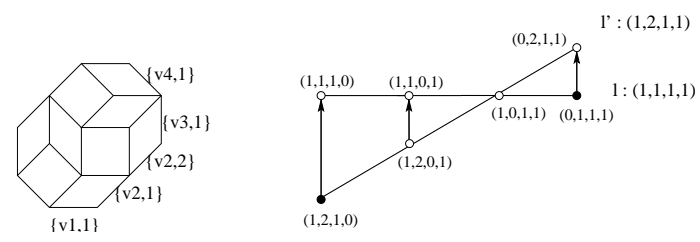

Fig. 3: a tiling and the associated diagram.

\section{Structures for lexicographic sequences of vectors}

We say that sequence $\left(v_{1}, v_{2}, \ldots, v_{D}\right)$ is lexicographic if for each line $l$, defined by a subsequence of $d+2$ vectors, the set of points of $l$ is ordered according to the lexicographic order of types. In codimension 2 , one can assume without loss of generality that the given sequence is lexicographic. This is not true in the general case, but there exists a lexicographic sequence for any value of the parameters $d$ and $D$. It suffices to take a sequence $\left(v_{1}, v_{2}, \ldots, v_{D}\right)$ such that, for each subsequence $\left(v_{i_{1}}, v_{i_{2}}, \ldots, v_{i_{d}}\right)$, the determinant $\operatorname{det}\left(v_{i_{1}}, v_{i_{2}}, \ldots, v_{i_{d}}\right)$ is positive One obtains such a sequence from an increasing sequence $\left(x_{1}, x_{2}, \ldots, x_{D}\right)$ of positive distinct reals numbers stating: $v_{i}=\left(1, x_{i}, x_{i}^{2}, \ldots, x_{i}^{d-1}\right)$. For each subsequence $\left(v_{i_{1}}, v_{i_{2}}, \ldots, v_{i_{d}}\right)$, we have $\operatorname{det}\left(v_{i_{1}}, v_{i_{2}}, \ldots, v_{i_{d}}\right)=\Pi_{1 \leq i<j \leq d}\left(x_{j}-x_{i}\right)>0$. In the unitary case, the order of the directed space of tilings is isomorphic to a higher Bruhat order (9).

Theorem 2 For tilings constructed on lexicographic sequence the order $<_{\text {flip }}$ is graded poset with a unique maximal element and a unique minimal element 
To prove it, we introduce some other arrows (the secondary arrows) as follows: let $l=\left(p_{1}, p_{2}, \ldots, p_{D+2}\right)$ be a line, such that the point $p_{1}$ is the point of lowest label. If $p_{1}$ is black in $T$, then, or each integer $i$ such that $1 \leq i<D+2$, we have a secondary arrow from $p_{i}$ to $p_{i+1}$. Otherwise, we have a secondary arrow from $p_{i+1}$ to $p_{i}$. Secondary arrows depend on the tiling $T$, at the opposite of the primary arrows introduced previously. They encode a sense for each line. The arrow constraint is still satisfied: there is no (secondary) arrow starting in a white point and finishing in a black point.

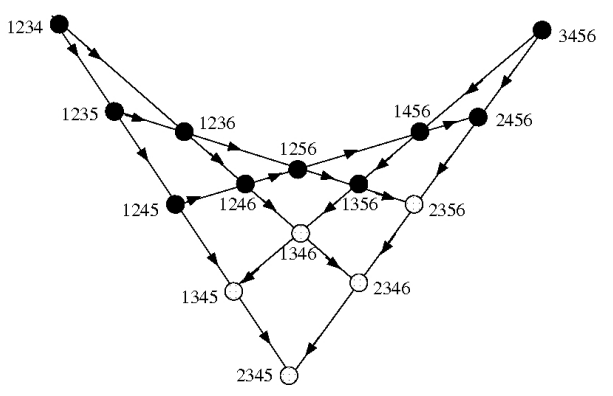

Fig. 4: orientation of lines for a tiling of a lexicographic unitary zonotope of codimension 3 and dimension 3 (non-null coordinates are given for each point).

Proposition 3 Let $T$ be a tiling. Each directed cycle of black points in the enriched diagram of $T$ is reduced to a single point.

The proof of this lemma is done by an precise induction, which involves some preliminary lemmas about relative dispositions of lines and arrows. We cannot give details here (see (6)). From this lemma, we easily deduce Th. 2 , since there exists a black point which can be turned in white without breaking constrains. This process can be repeated until there is no white point. In a symmetric way, in $T$, white points can be successively turned in black without breaking constraints.

\section{References}

[1] A. Björner, M. Las Vergnas, B. Sturmfels, N. White, G. M. Ziegler, Oriented Matroids, Encyclopedia of Mathematics 46, Cambridge University Press (1993),

[2] F. Chavanon, Aspects combinatoires des pavages, PhD. thesis LIP 2004-06, ENS Lyon, http: / / www.ens-lyon. fr/LIP/Pub/PhD2005.php

[3] F. Chavanon, E. Rémila, Rhombus tilings: decomposition and space structure, LIP research report, ENS Lyon RR2004-30, http: / / www. ens-1yon. fr/LIP/Pub/rr2004 . php.

[4] S. Felsner and H. Weil, A theorem on higher Bruhat orders, Discrete and Computational Geometry 23 (2003), 121-127.

[5] Richard Kenyon, Tiling a polygon with parallelograms, Algorithmica 9 (1993), 382-397. 
[6] E. Rémila, Structure of spaces of rhombus tilings in the lexicograhic case, LIP research report, ENS Lyon RR2005-19, http: / / www.ens-lyon.fr/LIP/Pub/rr2005.php.

[7] J. Richter-Gebert, G. Ziegler, Zonotopal tilings and the Bohne-Dress theorem, Contemporary Mathematics 178 (1994) 211-232.

[8] M. Widom, R. Mosseri, N. Destainville and F. Bailly, Arctic octahedron in three-dimensional rhombus tilings and related integer solid partitions, Journal of Statistical Physics 109 (2002) 945-965.

[9] G. Ziegler, Higher Bruhat orders and cyclic hyperplane arrangements, Topology 32 (1992), 259-279.

[10] G. Ziegler, Lectures on Polytopes, Graduate Texts in Mathematics, Springer-Verlag, 1995. 